\title{
Role of AGC1/aralar in the metabolic synergies between neuron and glia
}

\author{
Laura Contreras * \\ Centro de Biología Molecular Severo Ochoa CSIC-UAM, CIBER de Enfermedades Raras and Instituto de Investigación Sanitaria Fundación Jiménez Díaz, \\ Universidad Autónoma de Madrid, Madrid 28049, Spain
}

\section{A R T I C L E I N F O}

\section{Article history:}

Received 30 October 2014

Received in revised form 30 March 2015

Accepted 7 April 2015

Available online 15 April 2015

\section{Keywords:}

Aralar

Metabolism

Aspartate

Glutamine

Neuron

Astrocyte

\begin{abstract}
A B S T R A C T
Brain energetic requirements are elevated due to the high cost of impulse transmission and information storage, and are met mainly by glucose oxidation. The energy needs are closely matched by metabolic regulation, which requires the close cooperation of neurons and astrocytes and involves highly regulated fluxes of metabolites between cells. The metabolism in each type of cell is determined in part by its proteomic profile, which has been regarded as complementary. This review will consider the cellular distribution of the mitochondrial aspartate-glutamate carrier, aralar/AGC1/SLC25A12, and its role in the synergic metabolism between neurons and astrocytes.
\end{abstract}

(C) 2015 Elsevier Ltd. All rights reserved.
Neuroenergetic processes are essential for brain function. Brain's energy demand accounts for about $25 \%$ of total body glucose usage, even if brain represents approximately $2 \%$ of body weight. This is mainly caused by the high biosynthetic needs for synaptic plasticity and the stringent control of ionic homeostasis across cell membranes, which is essential to keep the necessary electrical properties for signal transmission, most of which is mediated by the neurotransmitter glutamate (Belanger et al., 2011). Neurons are the cells in charge of processing the information, encoded as electrical impulses, and therefore are considered the highest energy demanding cells in the brain. In fact, astrocytes were considered for a long time only as silent bystanders whose role was to support and nurture neurons (Navarrete and Araque, 2014). This view of astrocytes as second tier players has been challenged and reconsidered over the past decades with the recognition that astrocytes receive and integrate signals from neurons. Astrocytes thus actively respond to synaptic activity supporting the increase in metabolic demand associated with information integration and plasticity and releasing gliotransmitters (Perea et al., 2009), although the latter has recently been challenged (Fujita et al., 2014). In any event, neurons

Abbreviations: AGC, aspartate-glutamate carrier; GDH, glutamate dehydrogenase; MAS, malate-aspartate shuttle; NAA, $N$-acetyl aspartate; TCA, tricarboxylic acid cycle.

Tel.: +3491196 4651; fax: (+34) 911964420.

E-mail address: Icontreras@cbm.csic.es. and astrocytes need to act coordinately for the correct integration and storage of information, a cooperation based in transcellular fluxes of metabolites. Two aspects of the metabolic relationship have received great attention, the glucose utilization during brain stimulation and the glutamate/glutamine cycle.

Glucose is the main energy substrate for brain. Brain energy metabolism is highly oxidative, with an oxygen glucose index near to the theoretical maxima of six oxygen molecules per glucose molecule completely oxidized to $\mathrm{CO}_{2}+\mathrm{H}_{2} \mathrm{O}$ (Dienel, 2012a). However, during brain activation there is a mismatch between increases in cerebral metabolic rate of oxygen and glucose consumption, which returns a lowered oxygen glucose index by approximately $10 \%$, together with net efflux of lactate from the brain (Dienel, 2012a, $2012 b)$. Which cells are responsible for the increased lactate production? In vivo functional imaging of brain activity, although of invaluable usefulness for monitoring task evoked activation of regions and overall metabolism changes, lacks enough spatial resolution to answer the question. Therefore, the actual locus for lactate production, i.e. neuron or astrocyte, is a matter of debate, as lactate production has been reported to take place in both types of cells (Caesar et al., 2008; Mangia et al., 2009; Pellerin and Magistretti, 2013).

Glutamate is the main excitatory neurotransmitter, but has also other functions in the brain. Glutamate can also be used as energy fuel (McKenna, 2013) and it is the precursor of $\gamma$-aminobutyric acid (GABA), glutathione and other amino acids. Thus the metabolism of glutamate (and its derivates) has received much attention. It is 
widely accepted that, after synaptic release, astrocytes efficiently take up glutamate reducing its concentration in the cleft. In the astrocytes, glutamate may be converted into glutamine, which has no excitatory effect and therefore can be returned to neurons. There, glutamine will regenerate glutamate for neurotransmitter vesicle loading. This coordinated process between neurons and astrocytes is known as the glutamate-glutamine cycle and is considered central to glutamate homeostasis (Berl and Clarke, 1983; Daikhin and Yudkoff, 2000), although it is a simplified view of the process. In fact, the fate of glutamate taken up by astrocytes differs regarding its concentration as shown in astrocytic cultures where low glutamate concentration enhanced glutamine synthesis whereas larger glutamate concentration favored oxidative use of the amino acid in astrocytes (McKenna et al., 1996). In the neurons there may be also some carbon loss from the cycle due to oxidative metabolism of glutamine (Hertz, 2013; McKenna, 2013; Rothman et al., 2011). Because glutamate does not cross the blood-brain barrier (Hawkins et al., 1995), the loss of glutamate neurotransmitter has to be compensated by de novo synthesis of glutamine in astrocytes, which express both the anaplerotic enzyme pyruvate carboxylase and the glutamine synthetase (Norenberg and Martinez-Hernandez, 1979; Shank et al., 1985).

Fluxes between neurons and glia are central to their metabolic interaction and are sustained by diverse profile expression of transporters and enzymes involved. The varied expression of proteins or isoforms with different kinetic properties is relevant to determine the directionality of a cycle, the preferred energy fuel or the most likely pathway to take place within or between them. For example, the high affinity monocarboxylate carrier MCT2 is associated with nerve endings as opposed to low affinity isoforms MCT1 and MCT4 expression in astrocytes. This differential cellular distribution has been interpreted to reflect a neuronal preference for lactate as oxidative substrate when compared with astrocytes (Pellerin and Magistretti, 2013). However, it should be noted that both astrocytes and neurons can oxidize lactate for energy (McKenna et al., 1993). On the other hand, the differential expression between glutamine synthetase which is expressed only in astrocytes (Norenberg and Martinez-Hernandez, 1979) and glutaminase, which is expressed in both cell types but is more active in neurons (Cardona et al., 2014; Hogstad et al., 1988), together with distinct glutamate and glutamine uptake systems in astrocytes and neurons respectively, is determinant of the glutamate/glutamine cycle direction (Berl and Clarke, 1983; Daikhin and Yudkoff, 2000; Rothman et al., 2012).

Aralar, as member of the malate-aspartate shuttle, plays an important role in oxidative glucose metabolism, glutamine synthesis and glutamate oxidation. It has been reported to be expressed only in neurons, at least during postnatal development (Pardo et al., 2013, and references there-in) but this view is challenged by aralar mRNA being found in acutely isolated astrocytes (Lovatt et al., 2007). In any event, lower expression of aralar in glial cells would make them dependent on transcellular fluxes of neuronal born metabolites, like aspartate and $\mathrm{N}$-acetyl aspartate. Therefore, the presence of aralar in adult glia is still a matter of debate and should be studied because of its relevance for understanding the synergies between neurons and glia in norm and pathology.

\section{Cell localization of aralar, the brain specific aspartate-glutamate carrier}

Aralar/AGC1/SLC25A12 is the brain isoform of the mitochondrial aspartate-glutamate carrier (AGC). Aralar belongs to the superfamily of the mitochondrial carriers and participates in the malate-aspartate shuttle (MAS, Figure 1). Aralar and the AGC liver isoform citrin have $\mathrm{Ca}^{2+}$ binding motives in its amino terminal domain facing the intermembrane space that confer $\mathrm{Ca}^{2+}$ sensitivity (del Arco and Satrustegui, 1998; Satrustegui et al., 2007). Thus, transport and MAS activities are activated by extramitochondrial calcium increases below those promoting $\mathrm{Ca}^{2+}$ uptake through the calcium uniporter (Contreras et al., 2007; Pardo et al., 2006). As an essential member of MAS, aralar will in part determine the extent of oxidative metabolism in the cellular type (neuron or astrocyte) where it is expressed (see discussion later). Aspartate efflux from its production site in mitochondria requires aralar (Pardo et al., 2013), and therefore aralar expression will determine if a cell can use endogenous aspartate or relies in transcellular flux of exogenous aspartate for its cytosolic use. This section will explore the current evidences for aralar cellular localization within the brain.

In culture, both neurons and astrocytes express aralar, as determined by western blot. Expression was lower in astrocytes than in neurons, where it is associated with an increase of aralar protein levels during in vitro neuronal development which was also observed in vivo (Ramos et al., 2003). Consistently, enriched cortical neurons from E15-16 embryos grown in Neurobasal medium with glucose as main oxidative substrate (85-90\% neuronal purity with some astrocyte contamination, Pardo et al., 2006) from a mouse model deficient in aralar exhibited profound metabolic disturbances, among which are the increase in lactate production and the decreased aspartate levels that can be traced back directly to the absence of aralar activity (Pardo et al., 2011). On the other hand, astrocytes in culture express aralar and the liver isoform of the AGC, citrin, which was not found in neuronal cultures (Ramos et al., 2003). Citrin is practically undetectable at the protein and mRNA levels in the brain, where it is restricted to a few neuronal clusters (Contreras et al., 2010; Ramos et al., 2003). Astrocytes cultured from aralar KO mice did not have the metabolic alterations observed in neuronal cultures, as expected if aralar had an actual role in glucose oxidative metabolism of cultured astrocytes, i.e. no changes in aspartate levels and neither alteration in lactate/pyruvate utilization ratios were observed in aralar deficient astrocytes (Pardo et al., 2011). Thus, expression of both AGCs, aralar and citrin, in cultured astrocytes, which is readily observed by western blotting (Ramos et al., 2003), should be regarded with caution as may be an effect of the partial de-differentiation suffered by cells in culture (Lindsay et al., 2014; Lodin et al., 1979) or even reflect the presence of undifferentiated progenitors that express aralar (Ramos et al., 2003). In general, cultured astroglia should be regarded as an astrocyte-like cell type and may not represent in all aspects their in vivo counterparts.

In the brain, in situ mRNA hybridization pattern pointed to neurons as the cells expressing aralar (Ramos et al., 2003). However, it could be argued that the in situ hybridization has poor spatial resolution. A compromise between tissue slices and cultured cells is to acutely isolate brain cells and separate them afterwards to obtain pure populations. This is possible nowadays with promoter directed expression of fluorescent proteins toward a specific cell types in vivo, followed by fluorescence activated cell sorting (FACS) of brain isolated cells. Using this approach, Lovatt et al. (2007) isolated cells from a mouse model expressing GFP or YFP under the control of GFAP and Thy-1 promoter respectively, and analyzed the RNA profile of the sorted populations. No differences were found for aralar at the mRNA level between acutely isolated neurons and glial cells, which were shown to have an active glucose oxidative metabolism, as would be expected if aralar is present to allow MAS activity (Lovatt et al., 2007). However, Cahoy et al. (2008), employing a different mouse model with EGFP under the S100B promoter to sort neurons (EGFP-) and astrocytes (EGFP+), found aralar preferentially expressed in neurons. Another work used translating ribosome affinity purification (TRAP) methodology to study the RNA profile of different brain cell populations and also found aralar mRNA in astrocytes, although in this article the highest expression was found in neuronal populations (Doyle et al., 2008). In the retina, microarray data revealed 100 -fold lower aralar expression in Müller cell as 


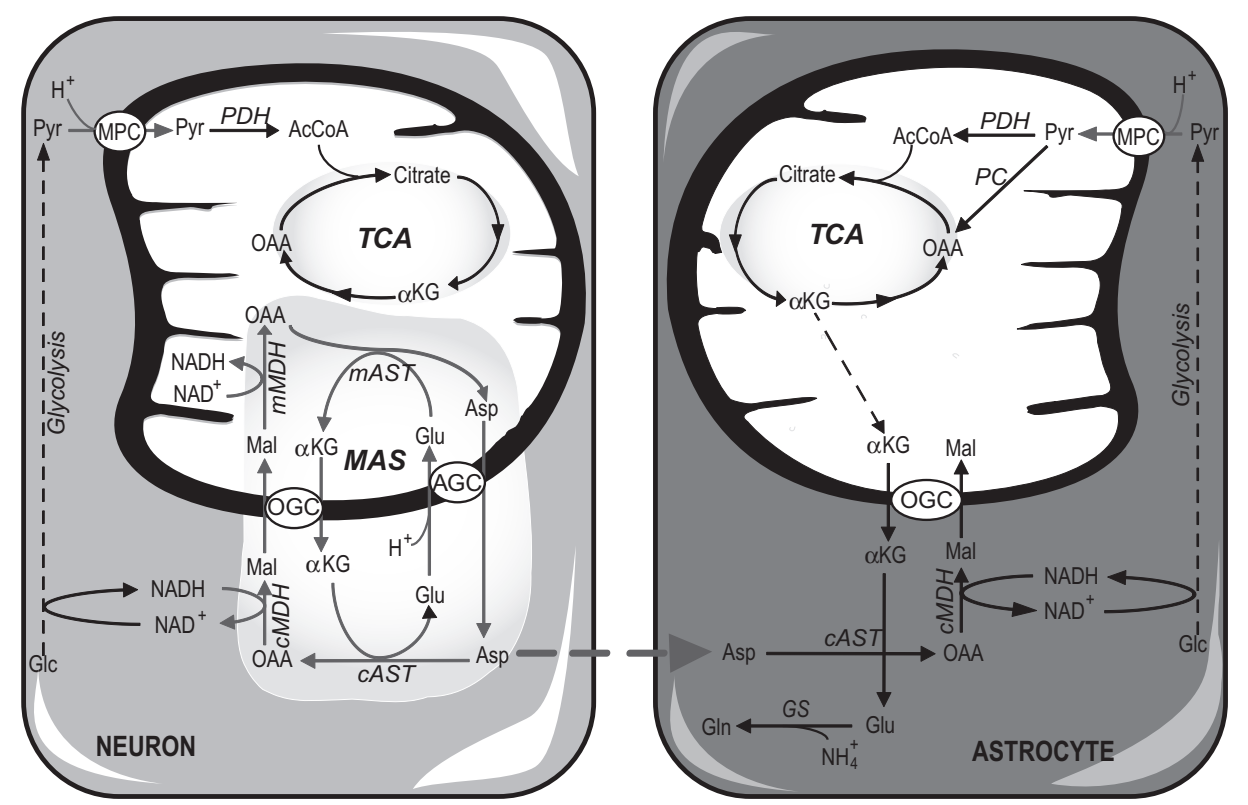

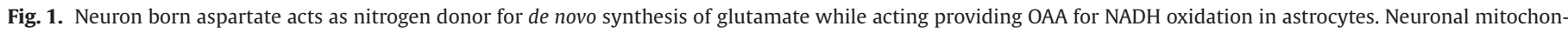

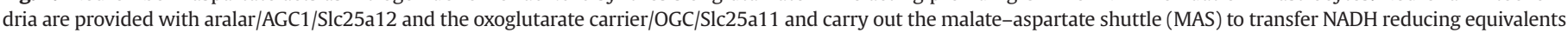

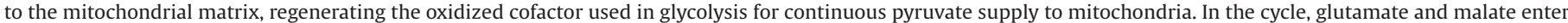

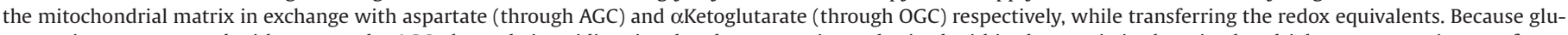

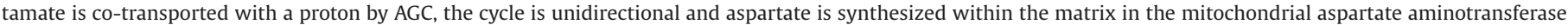

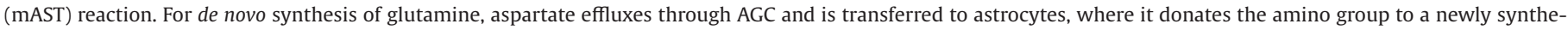

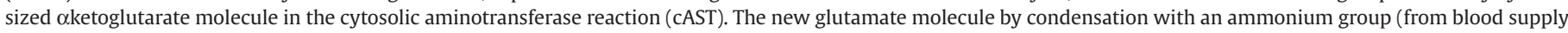

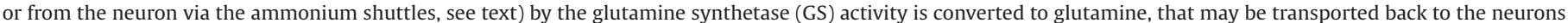

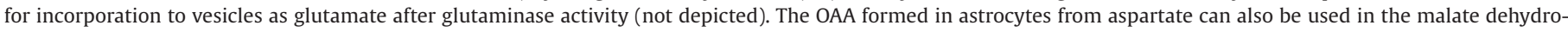

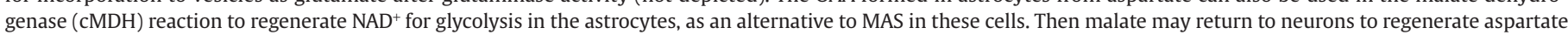

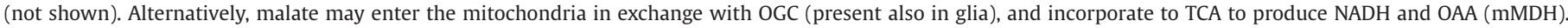

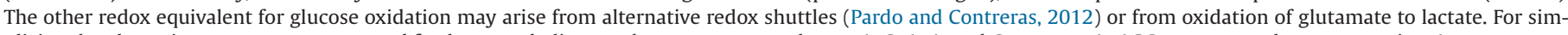

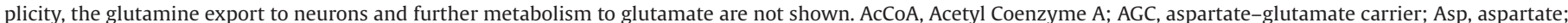

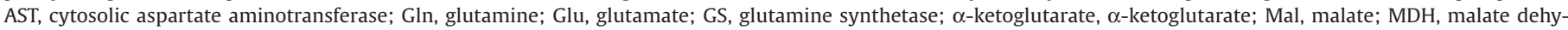

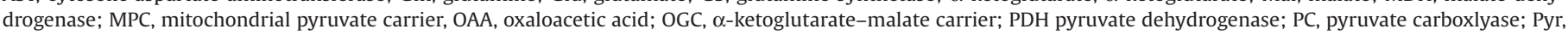
pyruvate, TCA, tricarboxylic acid cycle.

compared with photoreceptors (Roesch et al., 2008). Thus, in most of the studies aralar mRNA was found preferentially expressed in embryonic rat neurons or adult rat slices (Ramos et al., 2003), neurons from developing mice (Cahoy et al., 2008) or adult mice (Doyle et al., 2008). However, the preferential presence of aralar protein in neurons was also confirmed (Ramos et al., 2003) with antibodies raised against recombinant aralar (del Arco and Satrustegui, 1998).

Li et al. (2012) used a commercial monoclonal antibody that recognized no protein in liver extract, and reported similar aralar levels by western blot in acute isolated neurons and astrocytes from the same mouse model as Lovatt et al. (2007). Nonetheless, the absence of aralar from glia is in agreement with several results obtained by biochemical and inmunolabeling classical procedures. Previous work of immunohistochemical profiling by confocal microscopy found aralar in brain with a clear if not exclusive preference for neuronal populations (Berkich et al., 2007; Ramos et al., 2003). This distribution is re-enforced by biochemical data that support the idea of lower oxidative glucose metabolism in astrocytes compared with neurons (Belanger et al., 2011). A mitochondrial preparation without synaptosomes, and thus enriched in mitochondria from astrocytic origin, showed the expected enrichment in specific glial enzymes (pyruvate carboxylase, glutamine synthetase, mitochondrial branched-chain amino acid transaminase), but a significant decrease in aralar protein levels and aspartate production when compared with bulk mitochondria (Berkich et al., 2007). Aminooxyacetic acid, a general inhibitor of transminases and thus of MAS, severely affects lactate oxidation and tends to decrease glucose oxidation in synaptosomes, whereas in astrocytes the effect was completely the opposite supporting the conclusion of little shuttle activity (and aralar expression) in astrocytes (McKenna et al., 1993). In the retina, aralar has been also shown to be enriched in the photoreceptor cells and excluded from Müller cells, the resident glial cell type, by different specific antibodies (Lindsay et al., 2014; Xu et al., 2007) and by proteomic analysis of retina layers (Reidel et al., 2011). Müller cells exhibit elevated rate of aerobic glycolysis, with as little as $1 \%$ of glucose being transformed to $\mathrm{CO}_{2}+\mathrm{H}_{2} \mathrm{O}$ (Winkler et al., 2000). This fact is consistent with lack of a shuttle mechanism to regenerate redox power in the cytosol of Müller cells.

Finally, Pardo et al. (2011) showed at the electron microscopy level of resolution that aralar is present in neurons and not glia. Specifically, the analysis of electron microscopy images of brain using a highly specific aralar antibody showed that the vast majority ( $\approx 94 \%)$ of labeled aralar were present in neurons, where they associated preferentially with mitochondria (Pardo et al., 2011). The residual labeling $(\approx 7 \%)$ has been claimed as a demonstration of aralar presence in astrocytes (Peng et al., 2013). However, the fact that the label was distributed stochastically between mitochondria and cytosol, and that it was similar to the noise label level obtained in parallel experiments with aralar deficient tissues, strongly backs up the notion of aralar absence in astrocytes (Pardo et al., 2011).

Why are these differences found? Data obtained at the RNA level should always be confirmed at the protein level, and when possible, with a functional assay, because of the lack of correlation between the levels of mRNA and protein (Peng et al., 2013). Another divergence point may be the antibody selection, which varies within 
the studies presented and may have different specificities or affinities. Furthermore, as antibodies may bind to different unspecific proteins in different tissues, care should be taken and appropriate controls carried out in parallel to rule out false positives, with use of knock out samples of the tissue of interest being the best choice (Bordeaux et al., 2010; Zhang et al., 2003). It also should be noted that different animal models were used among the literature, and therefore the genetic variation between strains should be taken into account (Keane et al., 2011). For example, different AGC levels between mouse strains have been reported by western blot of heart extracts (Contreras et al., 2007). Finally, the age of the animal at the time of investigation should be considered. Metabolism changes from neonatal to adulthood, with upregulation or downregulation of key participants of metabolic pathways taking place. For example, aralar enrichment in neurons during the neonatal period would favor the building up of glutamate/glutamine pools that takes place (Morken et al., 2014) providing the carbon for de novo synthesis while selectively using glucose in anaplerotic pathways in the astrocytes (will be discussed later). In summary, due to the pivotal role of the aralar in brain metabolism, its localization should be confirmed beyond doubt in future studies.

The following sections will discuss the role of aralar in brain metabolism and in the metabolite fluxes between neuron and astrocytes.

\section{Role of aralar in glucose oxidation}

Brain cells use almost exclusively glucose as energy substrate, ketone bodies being the alternative during development and starvation (Belanger et al., 2011; Nehlig, 2004). After glycolysis, pyruvate generated in the cytosol is transported to the mitochondrial matrix where it is converted to acetyl-CoA for complete glucose oxidation at the tricarboxylic acid cycle (TCA, Fig. 1). Glycolysis also generates $\mathrm{NADH}$, and the oxidized cofactor in the cytosol needs to be regenerated to avoid glycolysis block at the glyceraldehydephosphate dehydrogenase step. Mitochondria are impermeable to both $\mathrm{NAD}^{+}$and NADH, and thus cells have evolved mechanisms to shuttle redox equivalents into the mitochondrial matrix. In brain, MAS is considered the main shuttle system as there is controversy upon the actual presence of a working glycerol-3-phospahte shuttle in brain cells. Redox balance can also be regained with the help of alternative (and minor) redox shuttles, and by reduction of pyruvate to lactate, but no redox power is transferred to mitochondria in this situation which is less efficient from an energetic point of view (Pardo and Contreras, 2012).

The prominent role of aralar, as part of MAS, in neurons is evident from the phenotype of the aralar deficient neurons, which have decreased respiration levels with glucose as fuel (Llorente-Folch et al., 2013). Furthermore, neurons lacking aralar are unable to regulate their respiration rates in response to $\mathrm{Ca}^{2+}$ transients, which would compromise the adequate response to energetic demands during stimulation (Llorente-Folch et al., 2013).

Astrocytes have reduced oxidative glucose metabolism (Belanger et al., 2011; McKenna et al., 1993), which may be considered a consequence of the low levels of shuttle activity due to little if not complete lack of aralar levels (see previous section). In cultured astrocytes, aerobic glycolysis was demonstrated to fuel the ATP necessary to efflux $\mathrm{Na}^{+}$after glutamate co-uptake from the synaptic cleft, providing a mechanism by which brain energy metabolism is tightly coupled to synaptic activity (Magistretti et al., 1999). The astroglial produced lactate was proposed to be exported to neurons for oxidative use (astrocyte-neuron lactate shuttle, ANLS) and lactate was shown to provide energy for synaptic transmission, a fact which seemed to support the hypothesis (Pellerin and Magistretti, 2013 for review). However, this shuttle hypothesis is based mainly on in vitro data, and has been challenged based on mathematical models and experimental data showing that neurons are also able to produce lactate during activity bursts, and thus may contribute also the net lactate efflux during brain activation (Caesar et al., 2008; Dienel, 2012a, 2012b; DiNuzzo et al., 2010a, 2010b; Gjedde and Marrett, 2001; Lindsay et al., 2014; Mangia et al., 2009). Recently, Patel et al. (2014) have demonstrated in vivo up-regulation of neuronal glucose metabolism as shown by increased glucose phophorylation, a fact that would also undermine the importance of astrocytic lactate as fuel to neurons in response to activation conditions. On the other hand, aerobic glycolysis in neurons which have robust MAS activity may seem paradoxical. However, MAS activity may become impaired by high $\mathrm{Ca}^{2+}$ concentrations as those that may take place during neuronal activity and that reach the mitochondrial matrix. Indeed, shuttle reconstitution with isolated mitochondria showed that intramitochondrial $\mathrm{Ca}^{2+}$ activation of $\alpha$ ketoglutaratedehydrogenase reduces efflux through the oxoglutarate carrier decreasing overall MAS activity, due to the competition of the two pathways, MAS and TCA, for a shared common metabolite, aketoglutarate (Contreras and Satrustegui, 2009). MAS inhibition by this mechanism has been proposed to take place in cultured neurons (Bak et al., 2012) and may divert pyruvate to lactate production (aerobic glycolysis) thus partially explaining the mismatch between glucose and $\mathrm{O}_{2}$ utilization upon brain activation (Dienel, 2012a), thus adding to other evidences in favor of lactate production by neurons and undermining the hypothesis of Magistretti and coworkers. It should be noted that MAS inhibition occurs at OGC level, which means that the AGC may remain active and contribute to the oxidative use of glutamate in a truncated TCA cycle (Yudkoff et al., 1994), as an alternative to impaired glucose oxidation. Importantly, isolated mitochondria from aralar deficient mice have reduced respiration with glutamate/malate but not with other respiratory fuels (Jalil et al., 2005) and therefore aralar is likely to play a role in the in vivo oxidation of glutamate.

In summary, aralar-MAS pathway is essential for proper glucose oxidative metabolism in neurons. The higher metabolic demand during brain activation leads to an increase in glucose metabolism with enhanced production of lactate whose site of production and further fate remains a matter of debate. The recent in vivo data presented by Patel et al. (2014) of increased glucose metabolism, together with demonstration of lactate production by neurons and the possibility of MAS inhibition during brain activity in vivo, question the central role of the astrocyte-neuron lactate shuttle, which may still be important in selected scenarios.

\section{Role of aralar in de novo synthesis of glutamate}

In the brain the glutamate/glutamine cycle is essential for efficient neurotransmission and glutamate homeostasis. However, the cycle is not stoichiometric and carbons are lost to oxidation (Hertz, 2013; McKenna, 2007). Indeed, part of the glutamate taken up by the astrocytes may be oxidized to satisfy the energetic demand of the uptake process and glutamine synthesis (Hertz, 2013; McKenna, 2013; Sonnewald et al., 1993) and thus become an alternative to the energy production pathway described previously (Magistretti et al., 1999). Glutamine can also be oxidized, probably in the neuron where the vast majority of glutaminase activity is present (Hogstad et al., 1988). Wherever the oxidation takes place, it drains $10-15 \%$ of cycle carbons, which is balanced by the anaplerotic synthesis of glutamate/glutamine in astrocytes (Hertz, 2013; Rothman et al., 2011). Net synthesis requires the specific glial enzyme pyruvate carboxylase to incorporate $\mathrm{CO}_{2}$ onto pyruvate to render a new molecule of oxaloacetate for condensation with acetyl-CoA and net synthesis of aketoglutarate, which is the direct precursor of the carbon skeleton of glutamate and therefore glutamine.

On the other hand, glutamine formation in astrocytes, either de novo or as part of the glutamate-glutamine cycle, requires at least one amino group, which is considered to be of neuronal origin (Bak 
et al., 2006; Rothman et al., 2012). Because simple rates of diffusion or $\mathrm{NH}_{4}$ from blood supply are unknown and may be too low and, in any case, $\mathrm{NH}_{4}$ would diffuse equally to astrocytes and neurons (Rothman et al., 2012), two mechanisms have been proposed to carry $\mathrm{NH}_{4}$ from neurons to astrocytes: alanine-pyruvate and the branchedchain amino acid shuttles (Bak et al., 2006; Daikhin and Yudkoff, 2000; Hutson et al., 2001; Waagepetersen et al., 2000). In these shuttles, $\mathrm{NH}_{4}$ produced in the glutaminase reaction (mainly in neurons) is fixed onto $\alpha$ ketoglutarate in the glutamate dehydrogenase (GDH) reaction. Then glutamate donates the ammonia group to pyruvate or $\alpha$ ketoacid in a transamination reaction, and the non neuroactive amino acid alanine or isoleucine/leucine formed is then transported toward astrocytes. The opposite reactions of transamination and GDH take place in astrocytes releasing $\mathrm{NH}_{4}$ for glutamine synthesis. The actual functionality of these shuttles is under debate for several reasons. (1) GDH $K_{m}$ would favor oxidation of glutamate in neurons, although it has been discussed that a local high $\left[\mathrm{NH}_{4}\right]$ generated by glutaminase activity may shift the equilibrium toward glutamate synthesis (Waagepetersen et al., 2000). (2) Specific genetic ablation of GDH in the central nervous system has little effect on synaptic transmission and glutamate pools (Frigerio et al., 2012), as would be expected if the alanine-pyruvate or branchedchain amino acid shuttles were essential for glutamine synthesis. (3) No correlation between alanine/pyruvate shuttle activity and the glutamate-glutamine cycle was revealed by repetitive stimulation of cultured granule cells (Bak et al., 2005). (4) Calculated flux of $\mathrm{NH}_{4}$ through the proposed shuttles is $3-5$ times lower to cover the demand of glutamine formation rates in astrocytes due to both glutamate-glutamine cycle and de novo glutamine synthesis (Rothman et al., 2012).

Based on the findings in a murine model deficient for aralar, Pardo et al. (2011) suggested neuron-born aspartate (or a derivate) as the metabolite acting as the $\mathrm{NH}_{4}$ carrier molecule (Figure 1). Aralar KO mouse has extremely reduced levels of aspartate, and its derivate $\mathrm{N}$-acetyl aspartate (NAA) (Jalil et al., 2005). Nuclear magnetic resonance studies with labeled $\left(1-{ }^{13} \mathrm{C}\right)$-glucose showed that de novo glutamine synthesis was impaired in the brain of the aralar KO mice, which explained the reduced levels of glutamate and glutamine in the brains of these knock out animals. Experiments with cultured astrocytes provided evidence that the defect was due to decreased neuronal-born aspartate, as (1) aspartate was reduced in cultured neurons from aralar KO mice, but no differences were observed in endogenous aspartate levels between wt and KO cultured astrocytes in basal conditions, pointing to the lack of an exogenous factor in vivo and (2) when supplemented in the incubation medium, aspartate increased glutamine levels to a greater extent than amino acids involved in the aforementioned $\mathrm{NH}_{4}$ shuttles (Pardo et al., 2011). These facts suggested that aspartate (or a related metabolite) produced by neurons may be required for glutamate (and glutamine) production in astrocytes in vivo, at least during the postnatal frame when experiments were performed.

The proposal of Pardo et al. (2011) has met some skepticism, mainly because so far no vesicular transporter for aspartate has been identified, as VGLUTs are specific for glutamate (Bellocchio et al., 2000; Fremeau et al., 2001, 2002). However, aspartate is present in synaptic vesicles and is released in a $\mathrm{Ca}^{2+}$ dependent way (Morland et al., 2013). Recently sialin, a sialic acid lysosomal carrier, was proposed to perform the uptake (Miyaji et al., 2008) but data obtained with the sialin KO mouse argue against this role for sialin, as aspartate uptake took place in the sialin $\mathrm{KO}$ mouse synaptic vesicles (Morland et al., 2013). Whichever the uptake system identity and release mechanism, aspartate efflux from neurons has been repeatedly observed and uptake into astrocytes demonstrated to take place by excitatory amino acid transporters (EAATs) with similar affinity to glutamate (Drejer et al., 1983; Rao and Murthy, 1993). Once in astrocytes, aspartate donates the $\mathrm{NH}_{4}$ to glutamate in a cytosolic transmination reaction. Labeling studies in cultured astrocytes show that the generated oxaloacetate either is converted into lactate through pyruvate recycling or enters the TCA for oxidation or glutamine generation (Bakken et al., 1997, 1998; Lindsay et al., 2014).

On the other hand, NAA, the acetyl donor for myelin synthesis, may act also as $\mathrm{NH}_{4}$-donor. $\mathrm{NAA}$ is produced in neurons in the condensation reaction of acetyl-CoA and aspartate (Ariyannur et al., 2010; Niwa et al., 2007; Wiame et al., 2010). Consistent with the decreased efflux of aspartate from mitochondria, NAA levels decrease in aralar KO, leading to hypomyelinization in mouse and human deficiency ("Global Cerebral Hypomyelination", OMIM \# 612949 (Falk et al., 2014; Jalil et al., 2005; Wibom et al., 2009)). NAA export to oligodendrocytes for myelin synthesis could also serve as $\mathrm{NH}_{4}$ transport, as cleavage of NAA releases aspartate that maybe used as amino donor in the transamination of $\alpha$ ketoglutarate to glutamate. However, aspartoacylase, the enzyme that break downs NAA releasing aspartate is present in oligodendrocytes (Mattan et al., 2010; Moffett et al., 2013) whereas both pyruvate carboxylase and glutamine synthetase have been reported to be absent (or low) in oligodendrocytes (Anlauf and Derouiche, 2013; Hertz, 2013). This enzymatic expression profile argues against de novo glutamate synthesis in oligodendrocites using the amoniun group transported by neuron-born NAA. Thus, aspartate released from NAA in oligodendrocytes by the aspartoacylase would still need to be exported to astrocytes in order to donate the amino group to the newly formed $\alpha$ ketoglutarate for efficient glutamine synthesis. Furthermore, the fact that NAA is a stable molecule with a low turnover ratio (Moffett et al., 2013) also argues against this metabolite being able to cover the ammonium demand for glutamine synthesis.

Although not shown in Fig. 1 (for the sake of simplicity), the proposed pathway requires obviously that carbons from aspartate are returned to neurons to avoid drainage of metabolites in that compartment. In that respect, the malate generated in the redox reaction may leave the astrocytic cytosol without entering the mitochondria as suggested by Pardo et al. (2013) or in the form of other TCA intermediate afterwards (Westergaard et al., 1994), and return to neurons for carbon replenishment. Alternatively, aspartate loss in neurons could be formed from essential amino acids or odd-chain fatty acids (Sonnewald, 2014) and to a lesser extent, by carboxylation of pyruvate by the mitochondrial malic enzyme (Hassel, 2000). In any event, the actual route for aspartate-carbon return to neurons has yet to be demonstrated.

In summary, the pathway proposed by Pardo et al. (2011) provides neuronal aspartate as source of ammonium groups for de novo synthesis of glutamate in brain astrocytes. In doing so, aspartate may also provide the astrocytic cytosol with means to transfer NADH/ $\mathrm{NAD}^{+}$redox potential to mitochondria. However, details on the stoichiometry of the pathway related to other important processes such as glutamate/glutamine oxidation, glucose oxidation rates and carbon balance within the brain demand further work.

\section{Role of aralar in retina metabolism}

Visual function starts with a complex biochemical cascade known as phototransduction in highly specialized retinal cells, the photoreceptors. Light stimulation initiates the cascade leading to hyperpolarization of photoreceptors and cessation of glutamate release, which is continuous in the dark (Punzo et al., 2012). Thus, retina metabolism is faster in the dark, and requires an excellent control of glutamate homeostasis (Bui et al., 2009). Besides the large ATP amounts to maintain membrane potential for neurotransmission, photoreceptors need to re-synthesize daily about $10 \%$ of their outer segments and are thus considered the highest energy consuming cells of the human body (Punzo et al., 2012). Not surprisingly, metabolic alterations are a common feature of retinitis pigmentosa 
a condition of visual loss due to photoreceptor degeneration with genetic cause (Punzo et al., 2012).

In a recent work, Du et al. (2013) studied the flux of ${ }^{13} \mathrm{C}$ labeled metabolites in the retina and found that glutamate, which is readily sequestered back into photoreceptors (Hasegawa et al., 2006), is protected from oxidation in a NADH/NAD dependent manner (Figure 2A). Flux of ${ }^{13} \mathrm{C}$-labeled metabolites in TCA was shown to be reduced by 100 -fold at the level of succinate. This means that about $98 \%$ oketoglutarate is protected from oxidation, likely leaving the cycle in exchange with cytosolic malate at the oxoglutarate carrier (Du et al., 2013). The oketoglutarate and glutamate pools are in fast equilibrium by the transaminase reaction (Mason et al., 1995), so protecting aketoglutarate from oxidation spares glutamate for neurotransmission or anabolism. Although $a$ priori, AGC1 would be irrelevant for $\alpha$ ketoglutarate efflux from mitochondria, its activity is mandatory to supply the cytosol with oxalacetate for malate production and $\alpha$ ketoglutarate exchange, if the cytosolic $\mathrm{NADH} / \mathrm{NAD}^{+}$ratio is adequate. The role of aralar may be inferred in aralar deficient retinas, where in spite of the elevated $\mathrm{NADH} / \mathrm{NAD}^{+}$ratio, measured as lactate/pyruvate, the ratio glutamate/succinate falls as no protection is possible (Du et al., 2013).

In spite of the protection mechanism, some glutamate may be oxidized and therefore re-synthesized in Müller cells. The aspartate role in glutamine de novo synthesis as amino group donor (Pardo et al., 2011) has been recently extended in the retina to also act as carbon source in an elegant pulse-chase study (Lindsay et al., 2014)
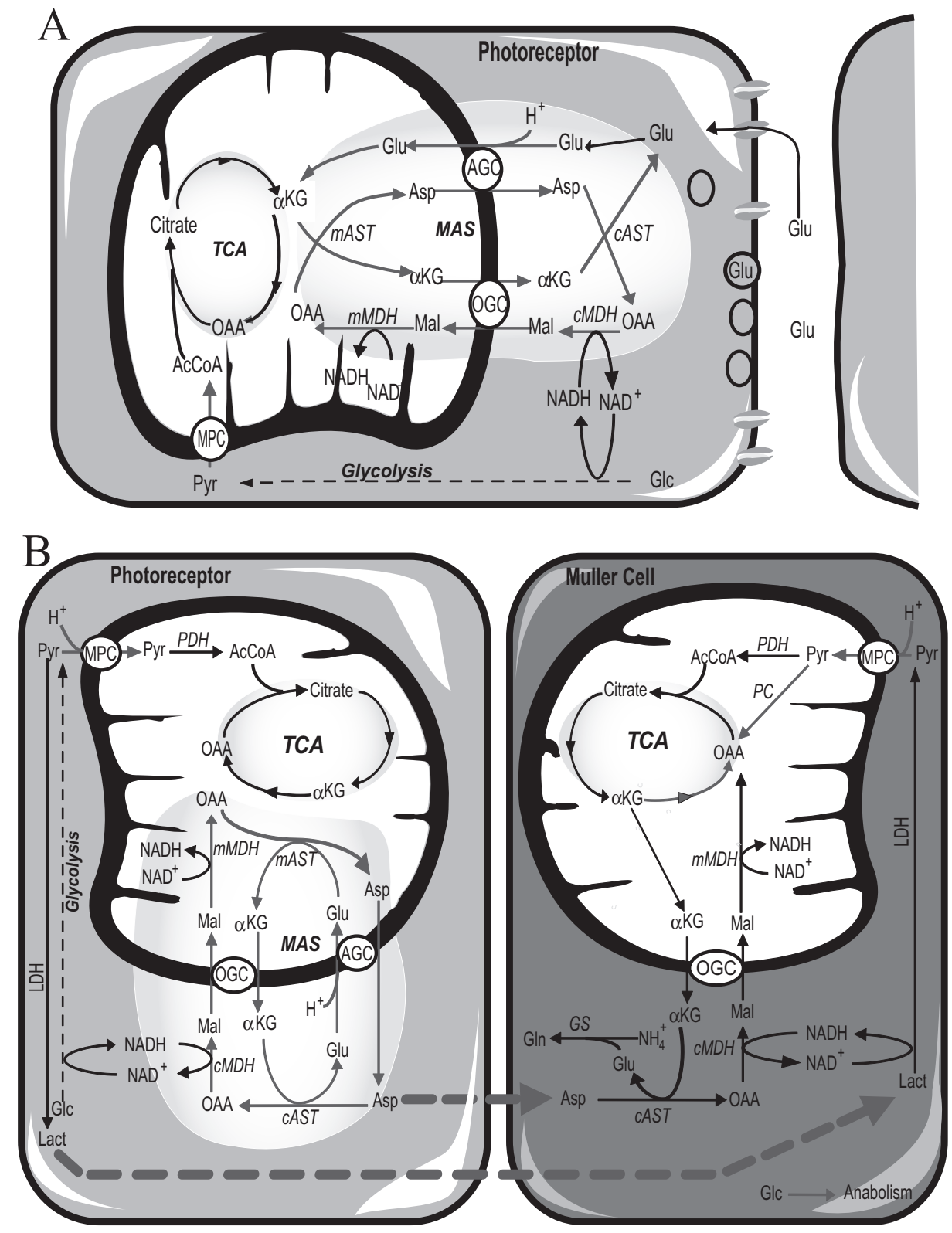

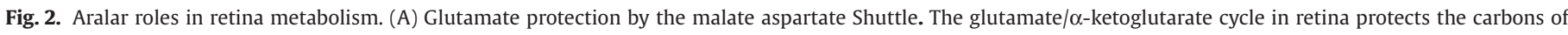

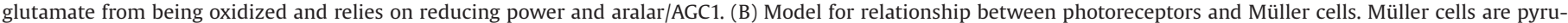

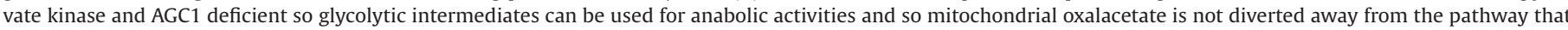

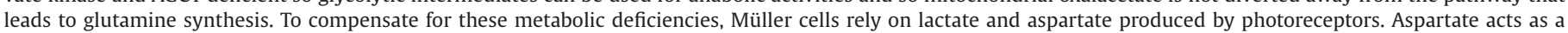

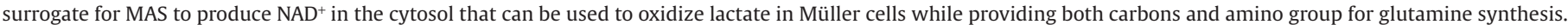

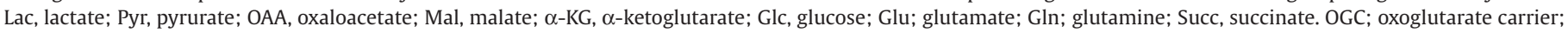
AGC, aspartate-glutamate carrier. Gray circles represent synaptic vesicles and gray forms represent glutamate transporters. 
(Figure 2). Glutaminase and glutamine synthetase activities are enriched in photoreceptors and Müller cells respectively (Roesch et al., 2008), similar to the neuron versus glia distribution in brain. Thus, ${ }^{13} \mathrm{C}_{5}$-glutamine was used to pulse-label metabolites first in photoreceptors and then to chase them in Müller cells. The pathway proposed involves de-amination to form ${ }^{13} \mathrm{C}_{5}$-glutamate which is metabolized to ${ }^{13} \mathrm{C}_{5}$ - $\alpha$ ketoglutarate and then to a low extent (see discussion earlier) to ${ }^{13} \mathrm{C}_{4}$ succinate, malate and oxalacetate in photoreceptors. ${ }^{13} \mathrm{C}_{4}$-aspartate arising from labeled oxalacetate in the TCA exits neuronal mitochondria through aralar and is transferred to Müller cells (Pardo et al., 2011). In Müller cells, ${ }^{13} \mathrm{C}_{4}$ aspartate acts as donor of $\mathrm{NH}_{4}$ for glutamate synthesis while providing oxalacetate for NADH oxidation by malate dehydrogenase. The further fate of aspartate labeled carbons can be traced along the TCA to ${ }^{13} \mathrm{C}_{3^{-}}$ $\alpha$ ketoglutarate, glutamate and glutamine (Lindsay et al., 2014). Based on the effect of aspartate uptake inhibition and exogenous aspartate degradation, it was concluded that the aspartate must be produced in photoreceptors, where most of glutaminase activity is located, and transferred to Müller cells (Lindsay et al., 2014). It should be noted that exogenous aspartate also recovered glutamine levels in aralar deficient retinae (Lindsay et al., 2014). As discussed previously for the brain, the pathway still needs to be refined in terms of mass balance between photoreceptor and Müller cells, especially regarding the source of carbon skeleton for aspartate replenishment in the photoreceptor.

Retinae exhibit a high aerobic glycolysis ratio, with $80-96 \%$ of glucose converted to lactate which is released to venous blood and to the inter-photoreceptor matrix (Ames et al., 1992; Du et al., 2013; Wang et al., 1997a, 1997b, 1997c). The site for lactate production was proposed to be the Müller cells, based on in vitro studies that kept in line with the astrocyte-neuron lactate hypothesis discussed earlier (Poitry-Yamate et al., 1995; Tsacopoulos and Magistretti, 1996). However, recent data oppose that view, reinforcing the idea that the lactate flux from astrocytes to neurons might not be as critical as it was thought to be (see Section 2). Specifically, pyruvate kinase absence from Müller cells, together with abundant expression of the M2 isoform of pyruvate kinase in photoreceptors and the extreme reduction in lactate production in retinae with no functional photoreceptors has led to the suggestion of photoreceptors as the major lactate producer cells in retina (Lindsay et al., 2014). Photoreceptor-born aspartate would also serve to regenerate $\mathrm{NAD}^{+}$in Müller cell's cytosol compensating for the little shuttle activity in Müller cells (Lindsay et al., 2014) (Fig. 2). Therefore, aspartate was shown to enhance the oxidative use of lactate in Müller cells, where glycolysis is severely decreased as no pyruvate kinase expression is likely (Lindsay et al., 2014; Roesch et al., 2008).

The lack of pyruvate kinase and AGC1 activities in Müller cells potentiates the anabolic pathways as opposed to energy production: the pentose phosphate pathway is primed over glycolysis and trapping of oxalacetate inside mitochondria primes glutamine synthesis. Although this may be an advantage for anabolic purposes, it also makes Müller cells dependent on neurons to provide metabolites that act as surrogates for pyruvate kinase and AGC activities (Lindsay et al., 2014; Roesch et al., 2008). Aspartate promotes oxidation of NADH to support lactate oxidation and provides both nitrogen and carbon for de novo glutamine synthesis.

\section{Conclusions}

Aralar/AGC1/Slc25a12 expression in brain is primarily and perhaps exclusively neuronal (at least in young animals), where it plays a pivotal role in neuroenergetics, as it is essential for efficient glucose and lactate oxidation, and for aspartate export to astrocytes to fuel de novo glutamine synthesis. In the retina, besides the aforementioned roles, aralar is involved in glutamate protection from oxidation in photoreceptors and in the export of photoreceptor aspartate for efficient lactate oxidation in Müller cells.

\section{Acknowledgements}

Dr. A. Franco, Dr. B. Pardo, Dr. A. del Arco and Dr. I.M. GonzálezGonzález are gratefully acknowledged for critical reading the manuscript and Prof. J. Satrústegui is acknowledged for helpful discussions. LC is recipient of a JAE-CSIC postdoctoral contract. No conflict of interest.

\section{References}

Ames, A., 3rd, Li, Y.Y., Heher, E.C., Kimble, C.R., 1992. Energy metabolism of rabbit retina as related to function: high cost of $\mathrm{Na}+$ transport. J. Neurosci. 12, 840-853.

Anlauf, E., Derouiche, A., 2013. Glutamine synthetase as an astrocytic marker: its cell type and vesicle localization. Front Endocrinol. (Lausanne) 4, 144.

Ariyannur, P.S., Moffett, J.R., Manickam, P., Pattabiraman, N., Arun, P., Nitta, A., et al., 2010. Methamphetamine-induced neuronal protein NAT8L is the NAA biosynthetic enzyme: implications for specialized acetyl coenzyme A metabolism in the CNS. Brain Res. 1335, 1-13.

Bak, L.K., Sickmann, H.M., Schousboe, A., Waagepetersen, H.S., 2005. Activity of the lactate-alanine shuttle is independent of glutamate-glutamine cycle activity in cerebellar neuronal-astrocytic cultures. J. Neurosci. Res. 79, 88-96.

Bak, L.K., Schousboe, A., Waagepetersen, H.S., 2006. The glutamate/GABA-glutamine cycle: aspects of transport, neurotransmitter homeostasis and ammonia transfer. J. Neurochem. 98, 641-653.

Bak, L.K., Obel, L.F., Walls, A.B., Schousboe, A., Faek, S.A., Jajo, F.S., et al., 2012. Novel model of neuronal bioenergetics: postsynaptic utilization of glucose but not lactate correlates positively with $\mathrm{Ca} 2+$ signalling in cultured mouse glutamatergic neurons. ASN Neuro. 4, pii: e00083.

Bakken, I.J., White, L.R., Aasly, J., Unsgard, G., Sonnewald, U., 1997. Lactate formation from [U-13C]aspartate in cultured astrocytes: compartmentation of pyruvate metabolism. Neurosci. Lett. 237, 117-120.

Bakken, I.J., White, L.R., Aasly, J., Unsgard, G., Sonnewald, U., 1998. [U-13C] aspartate metabolism in cultured cortical astrocytes and cerebellar granule neurons studied by NMR spectroscopy. Glia 23, 271-277.

Belanger, M., Allaman, I., Magistretti, P.J., 2011. Brain energy metabolism: focus on astrocyte-neuron metabolic cooperation. Cell Metab. 14, 724-738.

Bellocchio, E.E., Reimer, R.J., Fremeau, R.T., Jr., Edwards, R.H., 2000. Uptake of glutamate into synaptic vesicles by an inorganic phosphate transporter. Science 289, 957-960.

Berkich, D.A., Ola, M.S., Cole, J., Sweatt, A.J., Hutson, S.M., LaNoue, K.F., 2007. Mitochondrial transport proteins of the brain. J. Neurosci. Res. 85, 3367-3377.

Berl, S., Clarke, D.D., 1983. The Metabolic Compartmentation Concept. Alan R. Liss, Inc, New York, NY.

Bordeaux, J., Welsh, A., Agarwal, S., Killiam, E., Baquero, M., Hanna, J., et al., 2010. Antibody validation. Biotechniques 48, 197-209.

Bui, B.V., Hu, R.G., Acosta, M.L., Donaldson, P., Vingrys, A.J., Kalloniatis, M., 2009 Glutamate metabolic pathways and retinal function. J. Neurochem. 111, 589-599.

Caesar, K., Hashemi, P., Douhou, A., Bonvento, G., Boutelle, M.G., Walls, A.B., et al., 2008. Glutamate receptor-dependent increments in lactate, glucose and oxygen metabolism evoked in rat cerebellum in vivo. J. Physiol. 586, 1337-1349.

Cahoy, J.D., Emery, B., Kaushal, A., Foo, L.C., Zamanian, J.L., Christopherson, K.S., et al., 2008. A transcriptome database for astrocytes, neurons, and oligodendrocytes: a new resource for understanding brain development and function. J. Neurosci. 28, 264-278.

Cardona, C., Sanchez-Mejias, E., Davila, J.C., Martin-Rufian, M., Campos-Sandoval, J.A., Vitorica, J., et al., 2014. Expression of Gls and Gls2 glutaminase isoforms in astrocytes. Glia 63, 365-382.

Contreras, L., Satrustegui, J., 2009. Calcium signaling in brain mitochondria: interplay of malate aspartate NADH shuttle and calcium uniporter/mitochondrial dehydrogenase pathways. J. Biol. Chem. 284, 7091-7099.

Contreras, L., Gomez-Puertas, P., Iijima, M., Kobayashi, K., Saheki, T., Satrustegui, J. 2007. Ca2+ Activation kinetics of the two aspartate-glutamate mitochondrial carriers, aralar and citrin: role in the heart malate-aspartate NADH shuttle. J. Biol. Chem. 282, 7098-7106.

Contreras, L., Urbieta, A., Kobayashi, K., Saheki, T., Satrustegui, J., 2010. Low levels of citrin (SLC25A13) expression in adult mouse brain restricted to neuronal clusters. J. Neurosci. Res. 88, 1009-1016.

del Arco, A., Satrustegui, J., 1998. Molecular cloning of Aralar, a new member of the mitochondrial carrier superfamily that binds calcium and is present in human muscle and brain. J. Biol. Chem. 273 (36), 23327-23334.

Daikhin, Y., Yudkoff, M., 2000. Compartmentation of brain glutamate metabolism in neurons and glia. J. Nutr. 130, 1026S-1031S.

Dienel, G.A., 2012a. Brain lactate metabolism: the discoveries and the controversies. J. Cereb. Blood Flow Metab. 32, 1107-1138.

Dienel, G.A., 2012b. Fueling and imaging brain activation. ASN Neuro. 4, pii: e00093.

DiNuzzo, M., Mangia, S., Maraviglia, B., Giove, F., 2010a. Changes in glucose uptake rather than lactate shuttle take center stage in subserving neuroenergetics: evidence from mathematical modeling. J. Cereb. Blood Flow Metab. 30, 586-602. 
DiNuzzo, M., Mangia, S., Maraviglia, B., Giove, F., 2010b. Glycogenolysis in astrocytes supports blood-borne glucose channeling not glycogen-derived lactate shuttling to neurons: evidence from mathematical modeling. J. Cereb. Blood Flow Metab. 30, 1895-1904.

Doyle, J.P., Dougherty, J.D., Heiman, M., Schmidt, E.F., Stevens, T.R., Ma, G., et al., 2008. Application of a translational profiling approach for the comparative analysis of CNS cell types. Cell 135, 749-762.

Drejer, J., Larsson, O.M., Schousboe, A., 1983. Characterization of uptake and release processes for D- and L-aspartate in primary cultures of astrocytes and cerebellar granule cells. Neurochem. Res. 8, 231-243.

Du, J., Cleghorn, W., Contreras, L., Linton, J.D., Chan, G.C., Chertov, A.O., et al., 2013. Cytosolic reducing power preserves glutamate in retina. Proc. Natl. Acad. Sci. U.S.A. $110,18501-18506$

Falk, M.J., Li, D., Gai, X., McCormick, E., Place, E., Lasorsa, F.M., et al., 2014. AGC1 deficiency causes infantile epilepsy, abnormal myelination, and reduced N-acetylaspartate. JIMD Rep. 14, 77-85.

Fremeau, R.T., Jr., Troyer, M.D., Pahner, I., Nygaard, G.O., Tran, C.H., Reimer, R.J., et al., 2001. The expression of vesicular glutamate transporters defines two classes of excitatory synapse. Neuron 31, 247-260.

Fremeau, R.T., Jr., Burman, J., Qureshi, T., Tran, C.H., Proctor, J., Johnson, J., et al., 2002. The identification of vesicular glutamate transporter 3 suggests novel modes of signaling by glutamate. Proc. Natl. Acad. Sci. U.S.A. 99, 14488-14493.

Frigerio, F., Karaca, M., De Roo, M., Mlynarik, V., Skytt, D.M., Carobbio, S., et al., 2012. Deletion of glutamate dehydrogenase 1 (Glud1) in the central nervous system affects glutamate handling without altering synaptic transmission. J. Neurochem. $123,342-348$.

Fujita, T., Chen, M.J., Li, B., Smith, N.A., Peng, W., Sun, W., et al., 2014. Neuronal transgene expression in dominant-negative SNARE mice. J. Neurosci. 34, 1659416604.

Gjedde, A., Marrett, S., 2001. Glycolysis in neurons, not astrocytes, delays oxidative metabolism of human visual cortex during sustained checkerboard stimulation in vivo. J. Cereb. Blood Flow Metab. 21, 1384-1392.

Hasegawa, J., Obara, T., Tanaka, K., Tachibana, M., 2006. High-density presynaptic transporters are required for glutamate removal from the first visual synapse. Neuron 50, 63-74.

Hassel, B., 2000. Carboxylation and anaplerosis in neurons and glia. Mol. Neurobiol. $22,21-40$.

Hawkins, R.A., DeJoseph, M.R., Hawkins, P.A., 1995. Regional brain glutamate transport in rats at normal and raised concentrations of circulating glutamate. Cell Tissue Res. 281, 207-214.

Hertz, L., 2013. The glutamate-glutamine (GABA) cycle: importance of late postnatal development and potential reciprocal interactions between biosynthesis and degradation. Front Endocrinol (Lausanne) 4, 59.

Hogstad, S., Svenneby, G., Torgner, I.A., Kvamme, E., Hertz, L., Schousboe, A., 1988. Glutaminase in neurons and astrocytes cultured from mouse brain: kinetic properties and effects of phosphate, glutamate, and ammonia. Neurochem. Res. 13, 383-388.

Hutson, S.M., Lieth, E., LaNoue, K.F., 2001. Function of leucine in excitatory neurotransmitter metabolism in the central nervous system. J. Nutr. 131, 846S-850S.

Jalil, M.A., Begum, L., Contreras, L., Pardo, B., Iijima, M., Li, M.X., et al., 2005. Reduced $\mathrm{N}$-acetylaspartate levels in mice lacking aralar, a brain- and muscle-type mitochondrial aspartate-glutamate carrier. J. Biol. Chem. 280, 3133331339.

Keane, T.M., Goodstadt, L., Danecek, P., White, M.A., Wong, K., Yalcin, B., et al., 2011. Mouse genomic variation and its effect on phenotypes and gene regulation. Nature 477, 289-294.

Li, B., Hertz, L., Peng, L., 2012. Aralar mRNA and protein levels in neurons and astrocytes freshly isolated from young and adult mouse brain and in maturing cultured astrocytes. Neurochem. Int. 61, 1325-1332.

Lindsay, K.J., Du, J., Sloat, S.R., Contreras, L., Linton, J.D., Turner, S.J., et al., 2014. Pyruvate kinase and aspartate-glutamate carrier distributions reveal key metabolic links between neurons and glia in retina. Proc. Natl. Acad. Sci. U.S.A. $111,15579-15584$.

Llorente-Folch, I., Rueda, C.B., Amigo, I., del Arco, A., Saheki, T., Pardo, B., et al., 2013. Calcium-regulation of mitochondrial respiration maintains ATP homeostasis and requires ARALAR/AGC1-malate aspartate shuttle in intact cortical neurons. J. Neurosci. 33, 13957-13971, 13971a.

Lodin, Z., Faltin, J., Korinkova, P., 1979. The effect of dibutyryl cyclic AMP on cultivated glial cells from corpus callosum of 30-day-old rats. Physiol. Bohemoslov. 28, 105-111.

Lovatt, D., Sonnewald, U., Waagepetersen, H.S., Schousboe, A., He, W., Lin, J.H., et al., 2007. The transcriptome and metabolic gene signature of protoplasmic astrocytes in the adult murine cortex. J. Neurosci. 27, 12255-12266.

Magistretti, P.J., Pellerin, L., Rothman, D.L., Shulman, R.G., 1999. Energy on demand. Science 283, 496-497.

Mangia, S., I., Simpson, A., Vannucci, S.J., Carruthers, A., 2009. The in vivo neuron-toastrocyte lactate shuttle in human brain: evidence from modeling of measured lactate levels during visual stimulation. J. Neurochem. 109 (Suppl. 1), 55-62.

Mason, G.F., Gruetter, R., Rothman, D.L., Behar, K.L., Shulman, R.G., Novotny, E.J., 1995. Simultaneous determination of the rates of the TCA cycle, glucose utilization, alpha-ketoglutarate/glutamate exchange, and glutamine synthesis in human brain by NMR. J. Cereb. Blood Flow Metab. 15, 12-25.

Mattan, N.S., Ghiani, C.A., Lloyd, M., Matalon, R., Bok, D., Casaccia, P., et al., 2010 Aspartoacylase deficiency affects early postnatal development of oligodendrocytes and myelination. Neurobiol. Dis. 40, 432-443.
McKenna, M.C., 2007. The glutamate-glutamine cycle is not stoichiometric: fates of glutamate in brain. J. Neurosci. Res. 85, 3347-3358.

McKenna, M.C., 2013. Glutamate pays its own way in astrocytes. Front Endocrinol (Lausanne) 4, 191

McKenna, M.C., Tildon, J.T., Stevenson, J.H., Boatright, R., Huang, S., 1993. Regulation of energy metabolism in synaptic terminals and cultured rat brain astrocytes: differences revealed using aminooxyacetate. Dev. Neurosci. 15, 320-329.

McKenna, M.C., Sonnewald, U., Huang, X., Stevenson, J., Zielke, H.R., 1996. Exogenous glutamate concentration regulates the metabolic fate of glutamate in astrocytes. J. Neurochem. 66, 386-393.

Miyaji, T., Echigo, N., Hiasa, M., Senoh, S., Omote, H., Moriyama, Y., 2008. Identification of a vesicular aspartate transporter. Proc. Natl. Acad. Sci. U.S.A. 105, 1172011724.

Moffett, J.R., Arun, P., Ariyannur, P.S., Namboodiri, A.M., 2013. N-Acetylaspartate reductions in brain injury: impact on post-injury neuroenergetics, lipid synthesis, and protein acetylation. Front Neuroenergetics 5, 11.

Morken, T.S., Brekke, E., Haberg, A., Wideroe, M., Brubakk, A.M., Sonnewald, U., 2014. Neuron-astrocyte interactions, pyruvate carboxylation and the pentose phosphate pathway in the neonatal rat brain. Neurochem. Res. 39, 556-569.

Morland, C., Nordengen, K., Larsson, M., Prolo, L.M., Farzampour, Z., Reimer, R.J., et al., 2013. Vesicular uptake and exocytosis of L-aspartate is independent of sialin. FASEB J. 27, 1264-1274.

Navarrete, M., Araque, A., 2014. The Cajal school and the physiological role of astrocytes: a way of thinking. Front Neuroanat. 8, 33.

Nehlig, A., 2004. Brain uptake and metabolism of ketone bodies in animal models. Prostaglandins Leukot. Essent. Fatty Acids 70, 265-275.

Niwa, M., Nitta, A., Mizoguchi, H., Ito, Y., Noda, Y., Nagai, T., et al., 2007. A novel molecule "shati" is involved in methamphetamine-induced hyperlocomotion, sensitization, and conditioned place preference. J. Neurosci. 27, 7604-7615.

Norenberg, M.D., Martinez-Hernandez, A., 1979. Fine structural localization of glutamine synthetase in astrocytes of rat brain. Brain Res. 161, 303-310.

Pardo, B., Contreras, L., 2012. Redox shuttles in the brain. In: Choi, I.-Y., Gruetter, R. (Eds.), Advances in Neurobiology: Neural Metabolism In Vivo, vol. 4. Springer US, New York, pp. 841-883. doi:10.1007/978-1-4614-1788-0_29.

Pardo, B., Contreras, L., Serrano, A., Ramos, M., Kobayashi, K., Iijima, M., et al., 2006 Essential role of aralar in the transduction of small Ca2+ signals to neuronal mitochondria. J. Biol. Chem. 281, 1039-1047.

Pardo, B., Rodrigues, T.B., Contreras, L., Garzon, M., Llorente-Folch, I., Kobayashi, K., et al., 2011. Brain glutamine synthesis requires neuronal-born aspartate as amino donor for glial glutamate formation. J. Cereb. Blood Flow Metab. 31, 90-101.

Pardo, B., Contreras, L., Satrustegui, J., 2013. De novo synthesis of glial glutamate and glutamine in young mice requires aspartate provided by the neuronal mitochondrial aspartate-glutamate carrier aralar/AGC1. Front Endocrinol (Lausanne) 4, 149.

Patel, A.B., Lai, J.C., Chowdhury, G.M., Hyder, F., Rothman, D.L., Shulman, R.G., et al., 2014. Direct evidence for activity-dependent glucose phosphorylation in neurons with implications for the astrocyte-to-neuron lactate shuttle. Proc. Natl. Acad. Sci. U.S.A. $111,5385-5390$.

Pellerin, L., Magistretti, P.J., 2013. Sweet sixteen for ANLS. J. Cereb. Blood Flow Metab. 32, 1152-1166.

Peng, L., Guo, C., Wang, T., Li, B., Gu, L., Wang, Z., 2013. Methodological limitations in determining astrocytic gene expression. Front Endocrinol (Lausanne) 4, 176.

Perea, G., Navarrete, M., Araque, A., 2009. Tripartite synapses: astrocytes process and control synaptic information. Trends Neurosci. 32, 421-431.

Poitry-Yamate, C.L., Poitry, S., Tsacopoulos, M., 1995. Lactate released by Muller glial cells is metabolized by photoreceptors from mammalian retina. J. Neurosci. 15, 5179-5191.

Punzo, C., Xiong, W., Cepko, C.L., 2012. Loss of daylight vision in retinal degeneration: are oxidative stress and metabolic dysregulation to blame? J. Biol. Chem. 287, 1642-1648.

Ramos, M., del Arco, A., Pardo, B., Martinez-Serrano, A., Martinez-Morales, J.R., Kobayashi, K., et al., 2003. Developmental changes in the Ca2+-regulated mitochondrial aspartate-glutamate carrier aralar1 in brain and prominent expression in the spinal cord. Brain Res. Dev. Brain Res. 143, 33-46.

Rao, V.L., Murthy, C.R., 1993. Uptake and metabolism of glutamate and aspartate by astroglial and neuronal preparations of rat cerebellum. Neurochem. Res. 18, 647-654.

Reidel, B., Thompson, J.W., Farsiu, S., Moseley, M.A., Skiba, N.P., Arshavsky, V.Y., 2011. Proteomic profiling of a layered tissue reveals unique glycolytic specializations of photoreceptor cells. Mol. Cell. Proteomics 10 (M110), 002469.

Roesch, K., Jadhav, A.P., Trimarchi, J.M., Stadler, M.B., Roska, B., Sun, B.B., et al., 2008. The transcriptome of retinal Muller glial cells. J. Comp. Neurol. 509, 225238.

Rothman, D.L., De Feyter, H.M., de Graaf, R.A., Mason, G.F., Behar, K.L., 2011. 13C MRS studies of neuroenergetics and neurotransmitter cycling in humans. NMR Biomed 24, 943-957.

Rothman, D.L., De Feyter, H.M., Maciejewski, P.K., Behar, K.L., 2012. Is there in vivo evidence for amino acid shuttles carrying ammonia from neurons to astrocytes? Neurochem. Res. 37, 2597-2612.

Satrustegui, J., Pardo, B., Del Arco, A., 2007. Mitochondrial transporters as novel targets for intracellular calcium signaling. Physiol. Rev. 87, 29-67.

Shank, R.P., Bennett, G.S., Freytag, S.O., Campbell, G.L., 1985. Pyruvate carboxylase: an astrocyte-specific enzyme implicated in the replenishment of amino acid neurotransmitter pools. Brain Res. 329, 364-367.

Sonnewald, U., 2014. Glutamate synthesis has to be matched by its degradation where do all the carbons go? J. Neurochem. 131, 399-406. 
Sonnewald, U., Westergaard, N., Petersen, S.B., Unsgard, G., Schousboe, A., 1993. Metabolism of [U-13C]glutamate in astrocytes studied by 13C NMR spectroscopy: incorporation of more label into lactate than into glutamine demonstrates the importance of the tricarboxylic acid cycle. J. Neurochem. 61, 1179-1182.

Tsacopoulos, M., Magistretti, P.J., 1996. Metabolic coupling between glia and neurons. J. Neurosci. 16, 877-885.

Waagepetersen, H.S., Sonnewald, U., Larsson, O.M., Schousboe, A., 2000. A possible role of alanine for ammonia transfer between astrocytes and glutamatergic neurons. J. Neurochem. 75, 471-479.

Wang, L., Kondo, M., Bill, A., 1997a. Glucose metabolism in cat outer retina. Effects of light and hyperoxia. Invest. Ophthalmol. Vis. Sci. 38, 48-55.

Wang, L., Tornquist, P., Bill, A., 1997b. Glucose metabolism in pig outer retina in light and darkness. Acta Physiol. Scand. 160, 75-81.

Wang, L., Tornquist, P., Bill, A., 1997c. Glucose metabolism of the inner retina in pigs in darkness and light. Acta Physiol. Scand. 160, 71-74.

Westergaard, N., Sonnewald, U., Schousboe, A., 1994. Release of alpha-ketoglutarate, malate and succinate from cultured astrocytes: possible role in amino acid neurotransmitter homeostasis. Neurosci. Lett. 176, 105-109.
Wiame, E., Tyteca, D., Pierrot, N., Collard, F., Amyere, M., Noel, G., et al., 2010. Molecular identification of aspartate $\mathrm{N}$-acetyl transferase and its mutation in hypoacetylaspartia. Biochem. J. 425, 127-136.

Wibom, R., Lasorsa, F.M., Tohonen, V., Barbaro, M., Sterky, F.H., Kucinski, T., et al. 2009. AGC1 deficiency associated with global cerebral hypomyelination. N. Engl. J. Med. 361, 489-495.

Winkler, B.S., Arnold, M.J., Brassell, M.A., Puro, D.G., 2000. Energy metabolism in human retinal Müller cells. Invest. Ophthalmol. Vis. Sci. 41, 3183-3190.

Xu, Y., Ola, M.S., Berkich, D.A., Gardner, T.W., Barber, A.J., Palmieri, F., et al., 2007. Energy sources for glutamate neurotransmission in the retina: absence of the aspartate/glutamate carrier produces reliance on glycolysis in glia. J. Neurochem. $101,120-131$.

Yudkoff, M., Nelson, D., Daikhin, Y., Erecinska, M., 1994. Tricarboxylic acid cycle in rat brain synaptosomes. Fluxes and interactions with aspartate aminotransferase and malate/aspartate shuttle. J. Biol. Chem. 269, 27414-27420.

Zhang, Y., Li, N., Caron, C., Matthias, G., Hess, D., Khochbin, S., et al., 2003. HDAC-6 interacts with and deacetylates tubulin and microtubules in vivo. EMBO J. 22, 1168-1179. 